\title{
The association of Fuchs's corneal endothelial dystrophy with axial hypermetropia, shallow anterior chamber, and angle closure glaucoma
}

John F Pitts, Jeffrey L Jay

\begin{abstract}
A series of 24 patients with Fuchs's dystrophy are presented in whom detailed clinical measurement showed an association with axial hypermetropia and shallow anterior chamber. In 14 of these patients one cornea had developed oedema, of which 11 had required penetrating keratoplasty. Comparison of these eyes with the fellow eyes without corneal oedema revealed that the anomalies in measurement were not due to the process of decompensation. These 14 patients were then compared with the remaining 10 patients without corneal oedema in either eye, and a similar profile of anomalous measurements was observed. The whole group of 24 patients were then compared with three separate control groups, and in each case a significant trend towards hypermetropia, short axial length, and shallow anterior chamber was noted. The mean spherical equivalent refractive error in the patients with Fuchs's dystrophy was $+2.48 \mathrm{D}$ compared with $-0.31 \mathrm{D}$ for controls; corresponding means for axial length were $22 \cdot 1$ $\mathrm{mm}$ compared with $23.4 \mathrm{~mm}$; and for anterior chamber depth were $2.2 \mathrm{~mm}$ compared with $2.7 \mathrm{~mm}$. Each of these differences was statistically significant, but there was no significant difference for the keratometry measurements between patients and controls. Five of $24(21 \%)$ of the patients had problems related to shallow anterior chambers of whom $3(12 \%)$ had manifest angle closure glaucoma requiring surgical peripheral iridectomy. The aetiology of Fuchs's dystrophy and of ametropia is discussed and possible modes of association outlined. This previously unrecognised association gives a rational basis for the widely accepted practice of combining penetrating keratoplasty with lens extraction and has several other practical implications which are important in the differential diagnosis and treatment of Fuchs's dystrophy and angle closure glaucoma.
\end{abstract}

Fuchs's endothelial dystrophy accounts for about $10 \%$ of penetrating keratoplasties in series conducted in Europe and the USA. ${ }^{12}$ Most authors advocate combined keratoplasty and lens extraction in this condition..$^{3-7} \mathrm{~A}$ few offer as an explanation an increased risk of angle closure glaucoma, ${ }^{258}$ and one goes as far as to state that the Fuchs's dystrophy patients tend to have shallow anterior chambers. ${ }^{9}$

It had been noted that most of the patients attending the external eye diseases clinic of the Tennent Institute of Ophthalmology with
Fuchs's endothelial dystrophy were hypermetropic and several had had associated complications such as acute angle closure glaucoma. We could find no published analysis of this possible association, though Bigar and Witmer ${ }^{10}$ reported an unusually high incidence of bilateral endothelial dystrophy in a series of patients who had sustained a unilateral attack of angle closure glaucoma. Fuchs himself ${ }^{11}$ did not comment on such an association, but he gives details of refraction on five of his 13 cases, and we have calculated a mean spherical equivalent of $+2.45 \mathrm{D}$ in these. We therefore carried out a retrospective study to determine if there was a significant association.

\section{Materials and methods}

All patients with Fuchs's dystrophy attending the External Eye Clinic were re-examined and the diagnosis confirmed in each case. Two categories of patient were identified:

(a) Patients with bullous keratopathy; most of these had required penetrating keratoplasty and the diagnosis had been confirmed histologically, but a few were still being managed conservatively.

(b) Patients without bullous keratopathy but with the diagnostic endothelial changes of Fuchs's dystrophy. Because of the controversy in classification of Fuchs's dystrophy as a distinct disease versus an extreme in a spectrum of age related change ${ }^{1213}$ our study required slit-lamp identification of confluent central cornea guttata both on retroillumination and specular reflection.

The following details were recorded for each patient: age; sex; spectacle refraction (past and present); age at first reading correction; history of amblyopia or childhood squint; history of angle closure glaucoma or peripheral iridectomy; keratometry; ultrasonic A-scan biometry of anterior chamber depth and axial length.

Spectacle Refraction. The current refraction. was recorded and compared with the past prescriptions obtained from opticians' records or focimeter readings of the patients' oldest distance spectacles. This was done to identify cases of progressive refractive error caused by changes in the crystalline lens with age.

Amblyopia, squint, angle closure glaucoma, peripheral iridectomy. Information was obtained from the case records and confirmed at the time of re-examination.

Keratometry. Corneal radius was measured with the Javal-Schiøtz keratometer and the mean value was used for analysis. Readings obtained after keratoplasty or cataract surgery and in eyes with corneal oedema were not used. 
Ultrasound biometry. Anterior chamber depth measurements were made with the Radionics Model 3000 Echo-Oculometer. Values following keratoplasty or cataract surgery and in eyes with corneal oedema were excluded. Axial length measurements were obtained with the same instrument. A mean of three readings was used providing the discrepancy between each reading did not exceed $0.1 \mathrm{~mm}$. Where the eye was aphakic or pseudophakic, the appropriate correction factor was applied.

Results for the study patients were compared with those for age and sex matched controls attending with other diagnoses. Controls were separately analysed in groups according to source, namely: (i) patients admitted for cataract surgery; (ii) a mixture of general ophthalmology clinic patients; (iii) patients with primary open angle glaucoma attending the glaucoma clinic. Patients with either Fuchs's dystrophy or angle closure glaucoma were excluded from each control group, as were those whose sole reason for attendance was refractive error. A total of 72 controls (24 in each group) was studied.

Eyes with corneal oedema or previous cataract extraction or keratoplasty were compared with their fellow eyes (paired $t$ test) to ensure that the process of corneal decompensation or surgery had not contributed to the development of hypermetropia and other features. The group of patients with corneal oedema or previous surgery was then compared with those without oedema in either cornea (unpaired $t$ test) to ensure that patients with and without corneal oedema were part of a uniform population which might then be treated as a single group for analysis. For this comparison the mean value for the two eyes of each patient was used unless only one result existed for a pair of eyes. This method of comparison is acknowledged to give the lowest
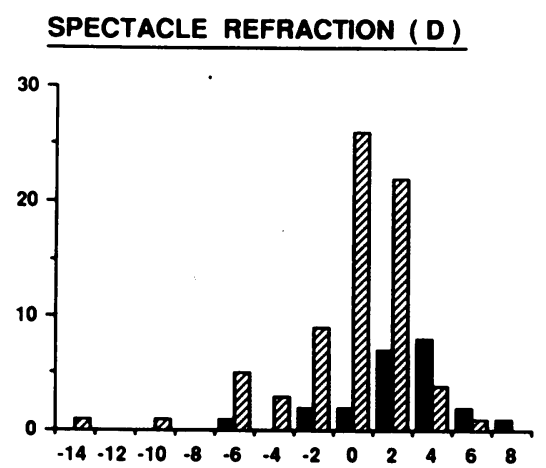

CONTROLS

ANTERIOR CHAMBER DEPTH (mm)

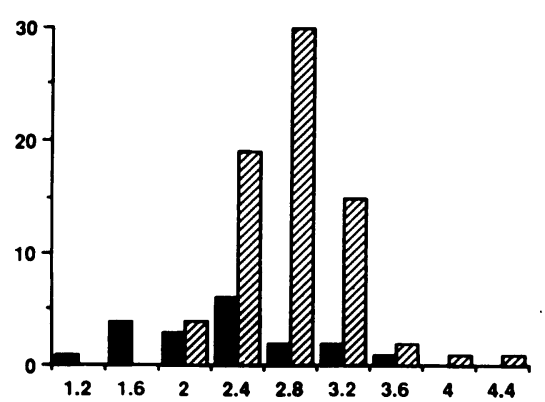

frequency of false positive associations and to have greater statistical precision than single eye comparisons or use of data from both eyes to double the sample size. ${ }^{14}$ A similar method was used in comparing Fuchs's dystrophy patients with controls. The whole group of 24 patients with Fuchs's dystrophy was then compared with each of the three control groups ( $t$ test) and with the combined control population by analysis of variance.

\section{Results}

There were 24 patients who fulfilled the criteria for diagnosis of Fuchs's dystrophy and who were available for re-examination. Fourteen had developed bullous keratopathy in one eye, and, of these, 11 had required a penetrating keratoplasty and three were being managed conservatively. The other 10 patients, although showing the diagnostic endothelial changes, had no corneal oedema in either eye. Both eyes of each patient fulfilled the diagnostic criteria, but not all measurements were available for all eyes, because keratoplasty, cataract extraction, or bullous keratopathy prevented readings valid for the intrinsic state of the eye. Therefore values for refraction ( 2 eyes), axial length ( 5 eyes), keratometry ( 15 eyes), and anterior chamber depth ( 20 eyes) remained undetermined.

Eyes with corneal oedema closely matched the fellow eyes of the same patients for refraction and axial length (paired $t$ test, $\mathrm{p}>0.5, \mathrm{n}=14$ for refraction and 11 for axial length). In addition the 14 patients with corneal oedema were compared with the 10 patients without corneal oedema ( $t$ test) with no significant difference for age $(0.2<\mathrm{p}<0.5)$, refraction $(0.2<\mathrm{p}<0.5)$, axial length $(p>0.5)$, and anterior chamber depth $(0.05<\mathrm{p}<0 \cdot 1)$. It seemed appropriate, there-

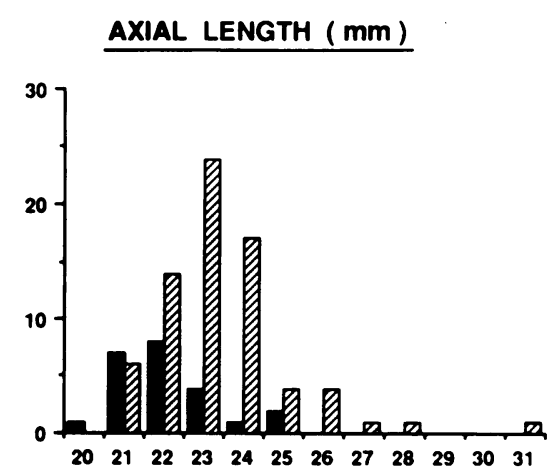

Figure 1 Histogram showing the refractive error, axial length, anterior chamber depth, and keratometry readings of 24 patients with Fuchs's dystrophy plotted alongside dystrophy plotted alon
72 control patients. 
SPECtaCle ReFraction (D)

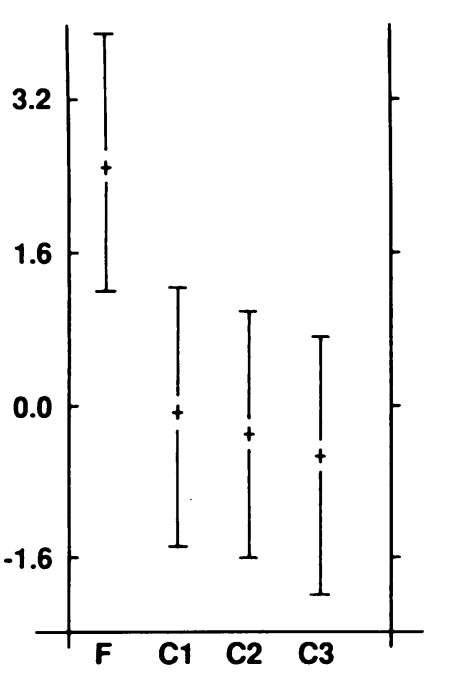

ANTERIOR CHAMBER DEPTH (mm)

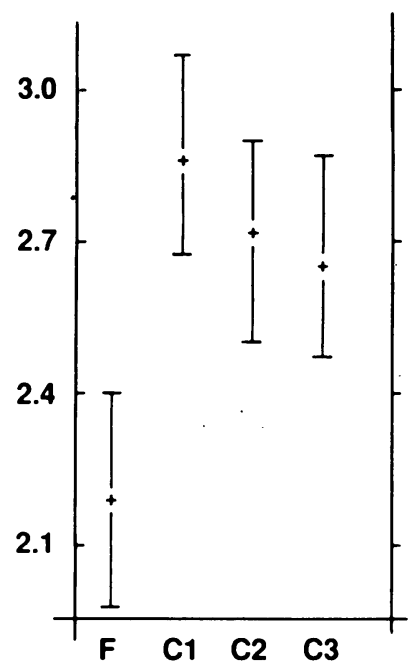

AXIAL LENGTH $(\mathbf{m m})$

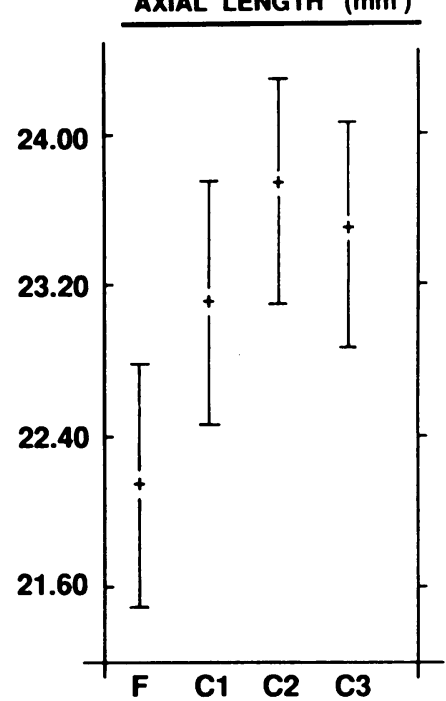

KERATOMETRY ( $\mathbf{m m})$

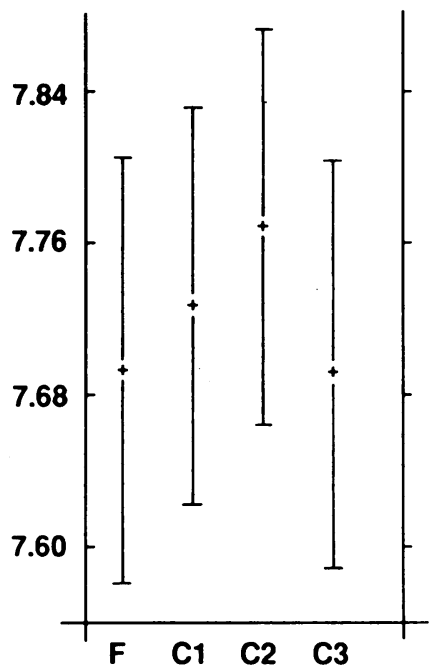

Figure 2 95\% Confidence intervals (mean \pm 2 SEM) for the refractive error, axial length, anterior chamber depth, and keratometry readings of 24 patients with Fuchs's dystrophy and 24 patients in each of 3 separate control groups. $F=F u c h$ s's dystrophy; $C 1=$ patients admitted for cataract surgery; $C 2=a$ mixture of general ophthalmology clinic patients; $C 3=$ patients with primary open angle glaucoma attending the glaucoma clinic.

fore, to conclude that all 24 patients could be treated as a single group for analysis.

Figure 1 is a histogram showing the Fuchs's dystrophy patients plotted alongside the combined control population. They appear more hypermetropic with shorter axial length and shallower anterior chambers, but similar keratometry values, compared with the controls. The 24 patients with Fuchs's dystrophy were then compared statistically with each of the three control groups and there was a significant difference in all measurements (unpaired $t$ test, $\mathrm{p}<0.001$ or $0.001<\mathrm{p}<0.01$ ) except keratometry $(\mathrm{p}>0.5)$. These comparisons are shown in Figure 2 with $95 \%$ confidence intervals (mean \pm 2 SEM).

In addition to the statistical evidence for an association a high prevalence of the three main complications of hypermetropia was detected in the Fuchs's dystrophy patients.

(i) Angle closure glaucoma. Five of $24(21 \%)$ had clinical problems related to shallow anterior chamber. Three of these $(12 \%$ of total) had spontaneous attacks of angle closure glaucoma requiring surgical iridectomy. While this cannot be compared with the control group (as angle closure glaucoma was excluded), it is well in excess of the quoted incidence of $0.5 \% .{ }^{15} 16$ The remaining two were noted to have a shallow anterior chamber following keratoplasty.

(ii) Amblyopia. Four of our 24 patients (17\%) had an amblyopic eye compared with two of the 72 controls $(3 \%)$.

(iii) Early presbyopia. Nine of 24 (38\%) of our patients with Fuchs's dystrophy required reading correction under the age of 30 years compared with nine of $72(12 \%)$ of the controls.

\section{Discussion}

The association of hypermetropia with shallow anterior chamber and angle closure glaucoma seems agreed. Tomlinson and Leighton ${ }^{17}$ found a mean anterior chamber depth of $2.31 \mathrm{~mm}$ in patients with primary angle closure glaucoma compared with $3.08 \mathrm{~mm}$ for controls; the corresponding mean axial lengths were 22.06 versus $22.58 \mathrm{~mm}$. Our results show that Fuchs's dystrophy is associated with axial hypermetropia and shallow anterior chamber, which provides a link with the increased risk of angle closure glaucoma.

It is interesting to speculate on the nature of the relationship between Fuchs's dystrophy and axial hypermetropia. It is unlikely that the endothelial dystrophy leads to axial hypermetropia, as hypermetropia is known to develop in early life while Fuchs's dystrophy tends to become manifest over the age of 50 . Nevertheless Lorenzetti et al $^{13}$ have demonstrated a surprisingly high incidence of cornea guttata in early life, and Rodrigues and others ${ }^{18}$ have shown by electron microscopy that the changes in the posterior collagenous layer of Descemet's membrane can be detected as early as the first two decades. Therefore this possibility cannot be excluded entirely. It is difficult to conceive a mechanism whereby axial hypermetropia might cause endothelial dystrophy, and indeed not all cases of Fuchs's dystrophy have this refractive error. Complications such as manifest or subclinical episodes of angle closure and effects of contact lens wear may aggravate the endothelial abnormality but are unlikely to be its cause. We believe that a causal relationship (in either direction) is improbable and that the association arises from a common factor giving rise to both conditions by genetic linkage.

The inheritance of Fuchs's dystrophy is not resolved, but the weight of evidence favours autosomal dominance with variable penetrance. ${ }^{719}$ The inheritance of refractive errors is equally controversial, with the most illuminating work on the subject being that of Sorsby, ${ }^{20}$ who considered emmetropia to be the result of co-ordinated regulation of the individual components of refraction. For example, in 107 emmetropic eyes $(0$ to $+0.50 \mathrm{D})$ he found an axial length of 22.3 to $26.0 \mathrm{~mm}$, the eyes being emmetropic because of automatic adjustment of other components of refraction, mainly the corneal curvature. In 255 eyes with ametropia 
within the range +6 to $-4 \mathrm{D}$, he found that 235 had axial length values within the range of the emmetropic group (22.3 to $26.0 \mathrm{~mm})$ and that these were ametropic because of failed adjustment, mainly in terms of corneal curvature; he calls this group the correlation ametropias. Another 46 ametropic eyes, with more than +6 or $-4 \mathrm{D}$, almost all had an axial length outside the range of $22 \cdot 3$ to $26.0 \mathrm{~mm}$; these are ametropic due to a single abnormal value, commonly the axial length; this group he terms the component ametropias. From twin and family studies he concludes that emmetropia and correlation ametropia are inherited in a polygenic manner, while component ametropia is most likely to be monofactorial.

If we assume that our patients were correlation ametropes, then it seems that the mechanism for regulating corneal curvature has failed. This might indicate either genetic linkage between the polygene system for corneal curvature and Fuchs's dystrophy or that the endothelium is somehow involved in regulating corneal curvature and that an inherited endothelial abnormality prevents this from occurring, the same inherited anomaly later becoming manifest as Fuchs's dystrophy. If on the other hand our patients are component ametropes, there may be genetic linkage between the (monofactorial) inheritance of an abnormal axial length and the (monofactorial) inheritance of Fuchs's dystrophy.

In fact of the 33 hypermetropic eyes in our patients $25(76 \%)$ have an axial length below $22 \cdot 3$ mm compared with only $29 \%$ in hypermetropic control eyes. While we accept that our axial length measurements cannot be directly statistically compared with Sorsby's, these findings are nevertheless strongly suggestive that a high proportion of our patients are what Sorsby terms component ametropes and that the second hypothesis of genetic linkage between two dominant genes is more likely.

While we have shown that Fuchs's dystrophy is frequently associated with hypermetropia and its risks, we cannot estimate from our data the frequency of Fuchs's dystrophy in hypermetropia. Bigar and Witmer ${ }^{10}$ noted that 35\% of patients with angle closure glaucoma had bilateral endothelial dystrophy. If this is so, it is highly significant, as it means that the patients who are subject to angle closure glaucoma tend to have an endothelium which is vulnerable to damage from the attack.

The association of Fuchs's dystrophy with axial hypermetropia has practical implications. Acute angle closure glaucoma and Fuchs's dystrophy may be confused as pain, glare, haloes, blurring of vision, and a shallow anterior chamber may be present in both conditions. Indeed mydriasis for examination or treatment of patients with Fuchs's dystrophy may precipi- tate angle closure in eyes with the shallowest anterior chambers.

The increased risk of shallow anterior chamber following penetrating keratoplasty for Fuchs's dystrophy justifies the practice of removing the crystalline lens during the operation. Waring et al have stated that these patients benefit from a posterior chamber intraocular lens, and we prefer the Pearce tripod lens, a rigid design which prevents the anterior chamber from becoming shallow postoperatively. ${ }^{21}$ With the increased frequency of hypermetropia, biometry to calculate the required power of intraocular lens is important, but keratometry may be impossible once bullous keratopathy has developed. Biometry should therefore be performed early in the course of the disease.

We thank Dr Donald Allan and Dr Gordon Murray for statistical advice and Susan Laing for preparing the illustrations.

1 Lang GK, Naumann GO. The frequency of corneal dystrophies requiring keratoplasty in Europe and the USA. Cornea 1987; 6: 209-11.

2 Waring GO III, Rodrigues MM, Laibson PR. Corneal dystrophies II. Endothelial dystrophies. Surv Ophthalmol 1978; 23 : $147-68$

3 Dohlman $\mathrm{CH}$, Hyndiuk RA. Subclinical and manifest corneal oedema after cataract extraction. In: Castroviejo, et al, eds. Symposium on the cornea. Trans New Orleans Acad Ophthalmol. St Louis: Mosby, 1972; chapter 17: 214-35.

4 Arentsen JJ, Laibson PR. Penetrating keratoplasty and cataract extraction: combined vs nonsimultaneous surgery. Arch Ophthalmol 1978; 96: 75-6.

5 Waring GO III, Bourne WM, Edelhauser HF, Kenyon KR. The corneal endothelium. Normal and pathologic structure and function. Ophthalmology 1982; 89: 531-90.

6 Polack FM. Glaucoma in keratoplasty. Cornea 1988; 7: 67-9.

7 Wilson SE, Bourne WM. Fuchs' dystrophy. Cornea 1988; 7 2-18.

8 Fine $M$. Round table discussions. In: Castroviejo $R$, et al, eds. Symposium on the cornea. Trans New Orleans Acad Ophthalmol. St Louis: Mosby, 1972: 253-93.

9 Rice NS. Keratoplasty in bullous keratopathy. In: Casey TA ed. Corneal grafting. London: Butterworths, 1972: chapter 10

10 Bigar F, Witmer R. Corneal endothelial changes in primary acute angle closure glaucoma. Ophthalmology 1982; 89: 596-9.

11 Fuchs E. Dystrophia epithelialis corneae. Graefes Arch Clin Exp Ophthalmol 1910; 76: 478-508.

12 Goar EL. Dystrophy of the corneal endothelium (cornea guttata) with a report of a histological examination. $A m \mathcal{F}$ Opttata) with a report of a histo

13 Lorenzetti DN, Uotila MH, Parikh N, Kaufman HE. Central cornea guttata, incidence in the general population. Am $\mathcal{J}$ Ophthalmol 1967; 64: 1155-8.

14 Newcombe RG, Duff GR. Eyes or patients? Traps for the unwary in the statistical analysis of ophthalmological studies. BrF Ophthalmol 1987; 71: 645-6.

15 Kolker A, Hetherington JJr. Becker-Schaffer's diagnosis and management of the glaucomas. 4th ed. St Louis: Mosby, 1976 183

16 Hitchings RA. Glaucoma. In: Miller S, ed. Clinical ophtha mology. London: Butterworth, 1987; chapter 11: 304-20.

17 Tomlinson A, Leighton DA. Ocular dimensions in the heredity of angle closure glaucoma. Br $\mathcal{f}$ Ophthalmol 1973 57: 475-86.

18 Rodrigues MM, Krachmer JH, Hackett J, Gaskins R, Halkias A. Fuchs' corneal dystrophy: a clinicopathological study of the variation in corneal oedema. Ophthalmology 1986; 93: the varia.

19 Krachmer JH, Purcell JJJr, Young CN, Bucher KD. Cornea endothelial dystrophy. A study of 64 families. Arch Ophthalmol 1978; 96: 2036-9.

20 Sorsby A. Biology of the eye as an optical system. In: Duane TD, ed. Clinical ophthalmology. Hagerstown: Harper and Row, 1984; 1: chapter 3.

21 Jay JL. Extracapsular lens extraction and posterior chamber intraocular lens insertion combined with trabeculectomy. Br J Ophthalmol 1985; 69: 487-90. 